\title{
THE RELATIONSHIP BETWEEN COLLISION HISTORY AND A COMPUTERIZED ASSESSMENT OF VISUAL AND COGNITIVE SKILLS IN A SAMPLE OF SCHOOL BUS DRIVERS
}

\author{
Kenneth C. Mills \\ Profile Associates \\ Chapel Hill, North Carolina, USA \\ E-mail: profiler@intrex.net \\ Robert C. Hubal \\ RTI International \\ Research Triangle Park, North Carolina, USA \\ E-mail: rhubal@rti.org \\ Brent T. Ward \\ RTI International \\ Research Triangle Park, North Carolina, USA \\ E-mail: bward@rti.org
}

\begin{abstract}
Summary: The objective of this study was to explore whether measures of visual and cognitive performance in a safe computerized driving environment were associated with collision involvement and the cost of collisions in a sample of professional motor vehicle operators. One hundred and nine (109) school bus drivers in a large metropolitan area were asked to take a 15-minute interactive computer-based driving assessment. The skills included visual target identification, scanning in four directions, divided-attention, reaction time, steering smoothness, false positive responses, and evasive maneuvers. An overall score validated in previous research summarized each driver's performance. Each driver's collision history over the last three years was then compared to the driving assessment scores. Collision data included collision type, frequency, and damage cost associated with each incident. Drivers with collisions $(n=27)$ were compared to drivers with no collisions $(n=82)$. Drivers with collisions had significantly lower overall scanning and steering smoothness scores than drivers without collisions. Drivers with collisions also had significantly higher braking and target false-positive scores, indicating disorientation. The total cost of collisions for the lower $40^{\text {th }}$ percentile test scores was $\$ 42,261$, whereas the cost for the upper $60^{\text {th }}$ percentile was $\$ 10,314$. The results indicate that drivers who are prone to become disoriented and overwhelmed in a high-demand computerized assessment were more likely to have had collisions on the road. The relationship between collision cost/incidence and test scores suggests that a sufficiently complex and rapidly paced computerized assessment has utility in identifying drivers who would benefit from remedial training.
\end{abstract}




\section{BACKGROUND}

It is not an easy task to establish a relationship between motor vehicle collision risk and objective measures of driving behavior (Marottoli et al., 1994; Massie, Campbell, \& Williams, 1995). Because collisions occur infrequently and unpredictably, both collision and behavioral measures have to be taken on a large sample of drivers. Using large samples requires a behavioral assessment that is easily and cost-effectively administered, and has been demonstrated to gather relevant information on driving skills. Fortunately, virtual assessment environments have the potential to measure complex, integrated perceptual and cognitive skills, even for rarely experienced events, and give the student contextual guidance and immediate feedback based upon performance (Field, et al., 1999).

A starting point in identifying drivers at risk for collisions, then, is to quantify visual and cognitive skills in a safe virtual environment while placing the driver under pressure to force mistakes, and then to compare virtual performance with on-the-road performance. Previous retrospective studies that looked back on driving after performance on a computerized assessment indicate that visual processing deficits are strongly associated with actual driving performance and a history of driving problems (Owsley et al., 1991). In a prospective study, older drivers with a $40 \%$ or greater impairment on a computerized assessment of the useful field of view (UFOV) were 2.2 times more likely to crash during a three-year follow up (Owsley, et al., 1998). There is also evidence that computerized assessments of visual and cognitive skills show a positive correlation to driving skills of police cadets on the track (Mills et al., 1999; Mills \& Hubal, 2001).

This study used a PC-based test with embedded selective and divided-attention tasks validated in previous studies against sedatives, stimulants and fatigue (Mills, Parkman, \& Spruill, 1997; Mills et al., 2001). In the current study, test events were filmed on a track and required the participants to respond to hazards integral to the driving scene. The test used continual-action driving sequences that placed high stimulus and response demands on the participant.

\section{METHODS}

Four out of 29 school districts (with a total of 1,700 drivers) in a large metropolitan region were equipped with PC-based assessment stations, each appended with commercially available offthe-shelf steering wheels and foot pedals. Drivers were given unlimited access to the simulation, although for this study, only the first 15-minute assessment for each driver was used. After a practice lap, scores from four successive, increasingly difficult levels of the test were averaged. The software recorded driving performance with 26 subscale scores that represented visual target identification, divided-attention responding, steering, braking, evasive maneuvers, and responses to radio calls (auditory discrimination). The software also partitioned scanning to four quadrants of the visual field (up, right, down and left). The software setup offered choices of school bus (used here), law enforcement, ambulance, and fire vehicles.

An overall score derived from the visual and divided-attention subscales was used to conduct comparisons with collision incidence and cost data. Previous research showed that the overall score was the best linear combination of test events related to drug effects and driving performance (Mills, Parkman \& Spruill, 1996; Mills, et al., 1999). 
The loss data included three years of incidents summarizing all collision losses by drivers in the four districts that volunteered to participate. Collision data were compiled by the insurance trust for the school district and recorded collision type, frequency, and costs. The primary comparison was between collision incidence (yes/no) and the overall score. The sample size was not adequate to test hypotheses about accident frequency/type and overall score.

The participants were 109 school bus drivers who ran through the test in their free time. One hundred of the drivers reported their age, with a mean of 46.83 years and a median of 46 years. The age range was 24 to 70 years with the ages normally distributed. On average, the drivers took the test two times, with a range of 1 to 12 sessions. There was incentive in that the driver with the highest score at the end of the competition would receive a prize: individual rewards of $\$ 75$ were offered for the best score in each district, and a \$250 reward was given for the best driver in the final competition between districts. The incentives were unlikely to have affected the current data because the winning scores came from other than the first tests.

Overall and subscale scores were normally distributed, allowing parametric comparisons between groups. Student t-tests were used to compare the collision and no-collision group scores.

\section{RESULTS}

There were no significant differences among three of the four districts in the cost/incidence of collisions. One district's volunteers $(n=12)$ had no collisions. The overall test score did not differ significantly among the four districts.

Twenty-seven drivers out of 109 (25\%) in the four districts had collision records. Four of the collisions did not have costs associated with them. The mean cost per collision for the 27 drivers was $\$ 1,947.25$, ranging from $\$ 0$ to $\$ 24,728.00$. There were no significant age differences between the collision and no-collision groups.

There was a significant difference on the overall score for those drivers who had collisions $(\mathrm{n}=$ 27) compared with those drivers who did not have collisions $(n=82)(t=2.74, p<.015)$. Figure 1 shows the overall score and the directional scanning scores for the collision and no-collision groups. The mean score for the entire sample was 2.83 , with upper and lower $95 \%$ confidence limits of 4.56 and 1.09. Drivers with collisions had significantly lower scores for right scanning $(\mathrm{t}=2.63, \mathrm{p}<.009)$, up scanning $(\mathrm{t}=8.38, \mathrm{p}<.005)$, and down scanning $(\mathrm{t}=7.13, \mathrm{p}<.009)$.

Reaction time (RT) scores did not differ between the two groups, suggesting that complex visual and cognitive skills relevant to safe driving — but not overall motor skills — were the primary influence on test scores.

The non-collision drivers showed significantly smoother steering scores during the test $(\mathrm{t}=2.39$, $\mathrm{p}<.019$ ). Figure 2 illustrates group differences for false-positive (i.e., unnecessary) responses to brake and visual stimuli. Drivers with collisions showed significantly higher unnecessary responses on both the brake pedal and hand responses to visual targets ( $\mathrm{t}$-brake $=3.55, \mathrm{p}<$ .0006 ; t-targets $=4.317, \mathrm{p}<.0001$ ). The incidence of unnecessary responses is an index of a driver's disorientation. Additional support for the collision group's disorientation comes from measures of evasive maneuvers and virtual "crashes" with oncoming vehicles. The drivers with 
collisions on the road tended to have more crashes and execute less effective evasive maneuvers during the test than drivers with no collisions (Figure 2).

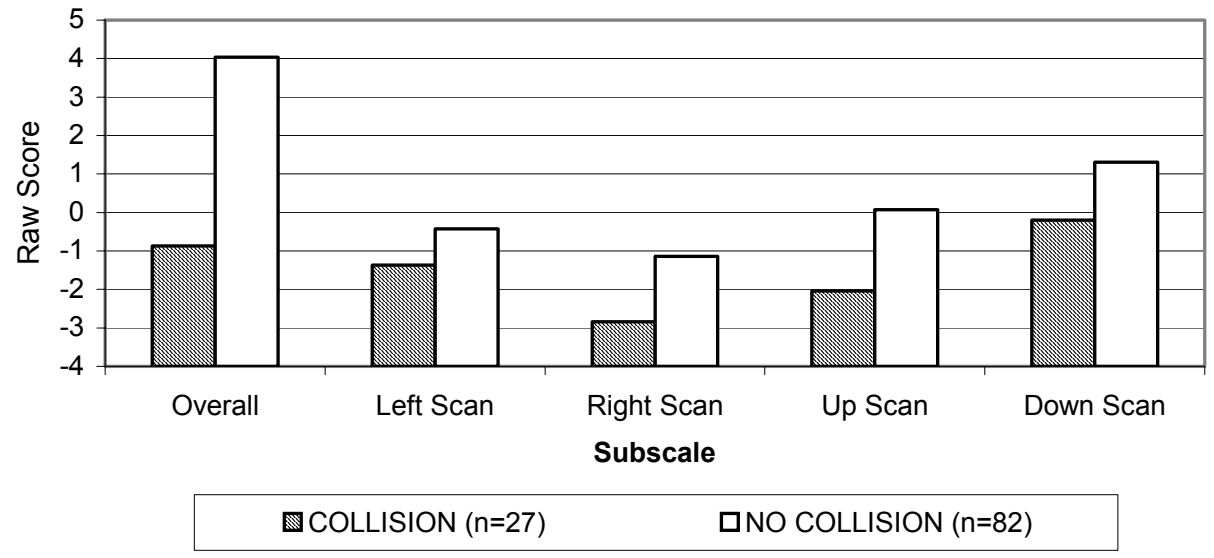

Figure 1. Overall score and directional scanning scores by collision and no-collision groups $(n=109)$

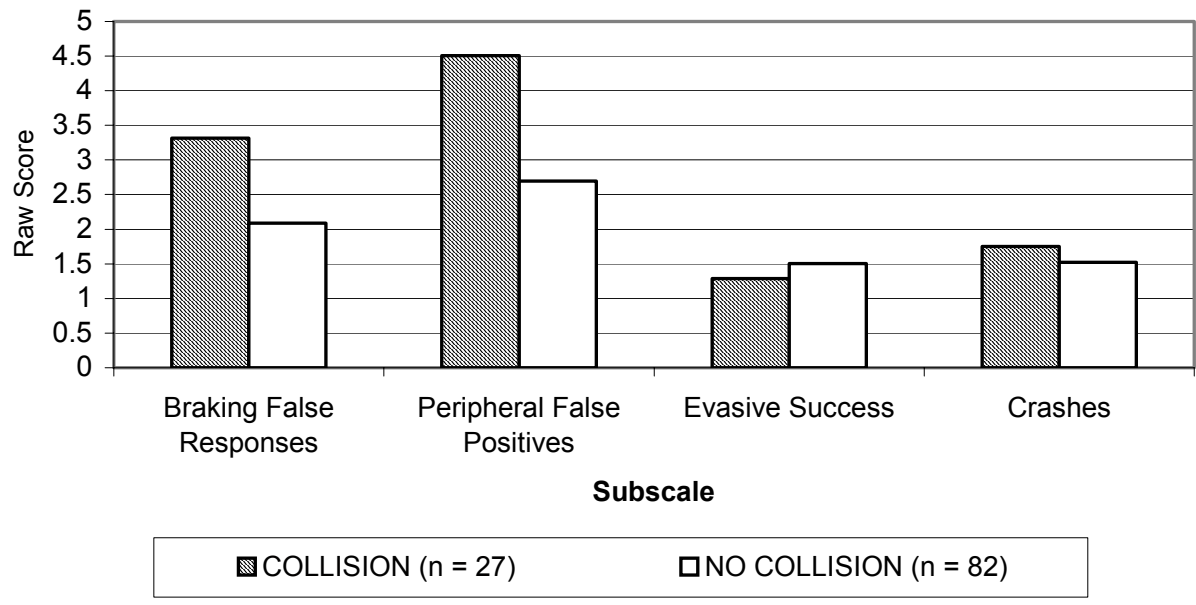

Figure 2. False positive and evasive action subscales by collision and no-collision groups $(\mathbf{n}=109)$

The test scores were converted to a frequency distribution that assigned all 109 drivers to five equal percentile categories: 1-20, 21-40, 41-60, 61-80, and 81-100. Quintiles were chosen because large employers often request a cutoff that identifies the lowest $20 \%$ of drivers. Figure 3 illustrates collision costs (left-hand y axis) and the mean value of overall scores (right-hand y axis) for each of the percentile categories. Figure 3 shows that collision costs were higher for the lower two percentile groups, and the mean overall score (as expected) increased linearly for the range of scores. Generally, positive scores represent calm, smooth performance whereas negative scores indicate jerky, overwhelmed driving. The correlation between collision cost and overall 
score $(n=23)$ was significant $(r=-0.51, p<.02)$, indicating that drivers with lower overall scores were more likely to have higher collision costs. Similar results were obtained when the scores were assigned to quartiles: $\$ 35,325$ for the lowest quartile, $\$ 4,185$ for the upper quartile.

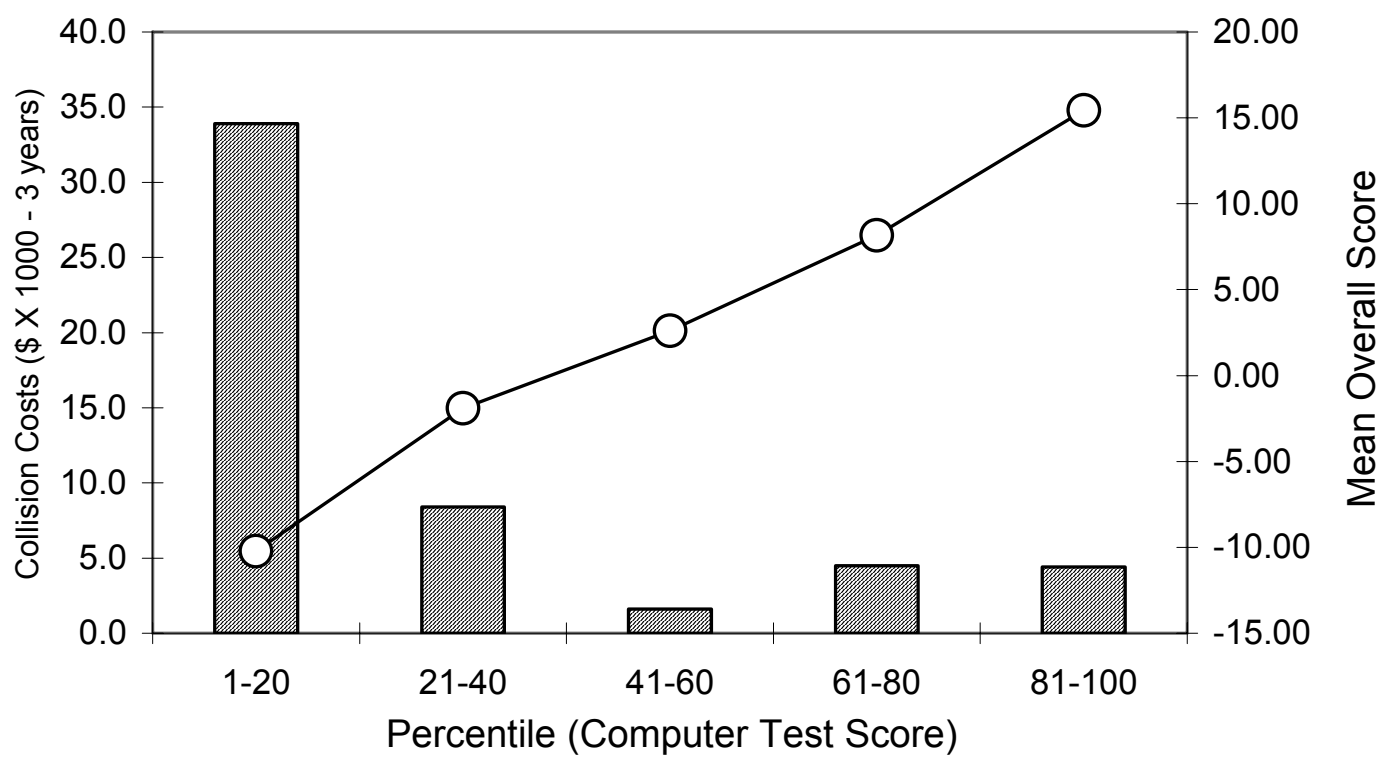

Collision Cost -0 -Mean Overall Score

Figure 3. Accident costs and overall score as a function of percentile ranking $(n=109)$

\section{CONCLUSIONS}

Drivers with a history of collisions were more likely than drivers with no collisions to become disoriented and overwhelmed when they were exposed to a brief (15 minute) yet complex interactive test that pushed visual and cognitive skills to the limit. It is well known in driver training that calm, smooth drivers perform and score better on track exercises. There is also an established link between computerized assessments and instructor ratings of driving performance under stressful conditions (Mills \& Hubal, 2001). The current study reinforces previous results that suggest a more long-term relationship between visual/cognitive skills and collision risk (Owsley et al., 1998). However, unlike previous research that tested elderly drivers, the present sample includes drivers who range in age from 24-70 and suggests that computerized visual/cognitive assessments may have utility for drivers of all ages. Whereas there were no age differences between the collision and no-collision groups, the overall scores showed a correlation with age of $\mathrm{r}=-0.47$, indicating that cognitive skills decline over the entire age range.

One possible limitation of the current study is that the sample of drivers from each school district consisted of volunteers. This might have introduced a bias in that drivers with deficits may have avoided taking the test. However, the mean cost of collisions for the drivers who volunteered to take the test was $\$ 1,947.25$, compared with $\$ 1,433.69$ for the remaining 1,700 drivers in the database for the districts. This would indicate that drivers who elected to take the test were a satisfactory population sample. 
Drivers with collisions had compromised visual search (scanning) during the simulation. The collision group showed significantly lower scores for scanning up, down and to the right. Similarly, while not significant, scanning scores to the left favored the non-collision group (Larsen \& Kines, 2002). On the road, scanning up includes awareness of the rear-view mirror (school bus passengers) and signal lights. Down scanning increases the driver's awareness of roadway hazards, crosswalks, and stop and turn lines. Right scanning covers traffic merges, bicyclists and pedestrians, and cross traffic. While not conclusive, the findings suggest that directional scanning deficits as a consequence of being overwhelmed may have contributed to previous accidents.

The results from this sample show that the visual and cognitive factors that may have contributed to collisions in the past remained unknown to the employer and the drivers. The results indicate that a formal assessment can identify drivers with specific deficits and suggest steps for remedial training. Importantly, a brief computerized assessment may provide a critical step in formulating a program to reduce the incidence and costs of motor vehicle collisions.

\section{ACKNOWLEDGEMENTS}

The authors wish to thank Ken Beres from MAISL for assisting in collecting the data and Tom Warren from RTI for reviewing the manuscript. This work was conducted using RTI internal research and development funds.

\section{REFERENCES}

Field, S.S., Frank, G.A., Helms, R.F., \& Hubal, R.C. (1999). Army learning \& training effectiveness symposium I: Individual learning. Final Report, March 30, Submitted to Battelle, RTP Office, Subcontract \# DAAH04-96-C-0086, Agreement \#99020, Delivery Order \#3066, Dated February 12, 1999.

Larsen, L., \& Kines, P. (2002). Multidisciplinary in-depth investigations of head-on and left-turn road collisions. Accident Analysis and Prevention, 34(3), 367-380.

Marottoli, R.A., Cooney, L.M., Jr., Wagner, D.R., Doucette, J., \& Tinetti, M.E. (1994). Predictors of automobile crashes and moving violations among elderly drivers. Annals of Internal Medicine, 121, 842-846.

Massie, D.L., Campbell, K.L., \& Williams, A.F. (1995). Traffic collision involvement rates by driver age and gender. Collision Analysis and Prevention, 21(1), 73-87.

Mills, K.C., Parkman, K.M., \& Spruill, S.E. (1996). A PC-based software test for measuring alcohol and drug effects in human subjects. Alcoholism: Clinical and Experimental Research, 20(9), 1582-1591.

Mills, K.C., Parkman, K.M., Smith, G.A., \& Rosendahl, F. (1999). Prediction of driving performance through computerized testing: High-risk driver assessment and training. Transportation Research Record, 1689, 18-24.

Mills, K.C. (2000). Tunnel Vision: Lifeline or Killer? Some Research on Why Training to "Keep Your Cool" is Valuable. Law and Order, 18(11), 80-83.

Mills, K.C., Spruill, S.E., Kanne, R.W., Parkman, K.M., \& Zhang, Y. (2001). Tunnel vision, stimulants, sedatives and fatigue: Risk factors for driving and piloting. Human Factors, 43(2), 310-327. 
Mills, K.C., \& Hubal, R.C. (2001). Correlational Data that Support a Constructive Assessment of Driving Skills. Proceedings of the $23^{\text {rd }}$ Interservice/Industry Training, Simulation, and Education Conference, Orlando FL.

Owsley, C., Ball, K., Sloane, M.E., Roenker, D.L., \& Bruni, J.R. (1991). Visual/cognitive correlates of vehicle collisions in older drivers. Psychology of Aging, 6, 403-414.

Owsley, C., Ball, K., McGwin, G., Jr., Sloane, M.E., Roenker, D.L., White, M.F., \& Overley, E.T. (1998). Visual processing impairment and risk of motor vehicle crash among older adults. Journal of the American Medical Association, 279(14), 1083-1088. 